\title{
Entendiendo los factores que determinan la actividad física en el entorno escolar desde la perspectiva de los niños y niñas
}

\section{Understanding the factors that determine physical activity in the school environment from the children's perspective}

\author{
Carlos Álvarez Bogantes ${ }^{1}$ \\ Escuela de Ciencias del Movimiento Humano y Calidad de Vida Universidad Nacional. \\ ceab.03@gmail.com
}

\begin{abstract}
Resumen
Este estudio explora las percepciones de los niños sobre las barreras y los facilitadores para realizar actividad física durante los recreos, con el fin de ofrecer el insumo necesario para las autoridades e investigadores educativos para el desarrollo de intervenciones y de políticas que promuevan la actividad física en este grupo de edad en el entorno educativo. Métodos: Se utilizó un diseño cualitativo con grupos focales y entrevistas a profundidad. Los datos fueron recolectados a través de 6 grupos focales, con un total de 90 niños (50 niños y 40 niñas) de tercer grado y las tres maestras del grado. Resultados: Las barreras más frecuentemente identificadas por los niños y las niñas fueron: falta de apoyo social, matonismo, falta de espacio y barreras organizativas. El espacio para jugar y el tiempo fue una barrera para todos niños, generando conflicto y exclusión de participar en actividades físicas en las niñas y en parte de los niños. Conclusión: Los participantes perciben que los entornos escolares no favorecen la actividad física; sin embargo, siguiendo su naturaleza y necesidad de moverse, una gran mayoría encuentra las formas de llenar esa gran necesidad de jugar y divertirse. Con base en los resultados de este estudio, se recomienda promover la actividad física a través del receso escolar, considerando las barreras en el entorno natural, social, físico y organizativo.
\end{abstract}

Palabras clave: educación física, niños, recreo escolar, barreras ambientales, creencias

\begin{abstract}
The study explores children's perceptions regarding physical activity barriers and facilitators during recess in order to provide the necessary input for governmental authorities and educational researchers to develop policies and interventions that promote physical activity for this age group within the educational environment. Methods: A qualitative design was employed that used focus groups and in-depth interviews for data collection. Data was collected from six focus groups, which included a total of 90 third-graders ( 50 boys and 40 girls) and their three teachers. Results: The most frequent barriers identified by children were: a lack of social support, bulling, a lack of space, and organizational barriers. A lack of space and time was a barrier for all children, resulting in conflict and exclusion from physical activities for girls and some of the boys. Conclusion: Participants perceive the school environment as not favorable for physical activity; however, following their nature and need to move, a large majority finds ways to fill that need to play and have fun. Based on the results of this study, physical activity should be promoted during recess, taking into consideration barriers in the natural, social, physical and organizational environments.
\end{abstract}

Keywords: physical activity, physical education, children, school recess, environmental barriers, beliefs 
Carlos Álvarez Bogantes

Revista en Ciencias del Movimiento Humano y Salud

URL: http://www.revistas.una.ac.cr/mhsalud

\section{Introducción}

Los beneficios físicos, mentales y sociales de la actividad física para los niños son ampliamente reconocidos (Floriani y Kennedy, 2008; Boreham y Riddoch, 2001; Wing et al., 2001). Sin embargo, a pesar de las recomendaciones de salud pública para que los niños realicen por lo menos 60 minutos de actividad física diaria de intensidad moderada a vigorosa (Strong et al, 2005), su no realización los expone a un mayor riesgo de desarrollar sobrepeso u obesidad (Biddle y Asare, 2011; Wareham, Van Siuijs y Ekelund, 2005) y muchas otras enfermedades como la diabetes y las enfermedades cardiovasculares (Buchan, Ollis, Thomas y Baker, 2012; Strong et al., 2005). Esto es especialmente preocupante cuando hablamos de los niños y niñas que presentan sobrepeso y obesidad, ya que la disminución de los niveles de actividad física desde la infancia es más evidente en ellos a través de la adolescencia (Janz, Burns y Levy, 2005). Por tanto, es importante, para hacer frente a la participación en actividad física durante la infancia, desarrollar estrategias de intervención desde la visión de los niños y niñas, con un acercamiento multinivel de la realidad (Buchan et al., 2012).

Con el fin de promover la actividad física entre los niños y niñas, varios estudios se han realizado en los entornos del hogar, la comunidad y la escuela (Jago, Baronowski, Zakeri y Harris, 2005; Van Sluij, McMinn y Griffin, 2007). En particular, la escuela ha sido identificada como una de las claves para la promoción de la actividad física de los niños (Ishii, Shibata, Sato y Koichiro 2014; Mota et al., 2005; Salmon y Timperio, 2007), especialmente durante los recreos escolares; sin embargo, se han reportado gran cantidad de barreras ambientales en el recreo, que se presentan como los factores con mayor peso para afectar la actividad física de los escolares desde la percepción de los niños y niñas (Ridgers, Salmon,Parrish, Stanley y Okely, 2012).

Se ha reconocido que para poder obtener el máximo provecho del entorno de los recreos escolares en la promoción de la actividad física de los niños y niñas, se requiere escucharlos y entender sus percepciones, creencias y actitudes hacia el movimiento humano (Pawlowski, Shipperijn y Troelsen, 2014). En relación con la percepción de los niños en cuanto a las barreras para realizar actividad física durante el recreo desde una perspectiva cualitativa, diferentes estudios han identificado como barreras importantes para la realización de actividad física durante el recreo: la falta de instalaciones y equipos, la intimidación o matonismo, la falta de políticas a nivel escolar, la ropa, la falta de apoyo de la maestra, la falta de espacio, el tiempo, la estética del patio, las habilidades físicas de los niños y la duración receso (Ishii, Sato, Shibata y Oka, 2013; Stanley, Boshoff y Dollman, 2012; Lake y Towshend, 2006). Esto indica que los ambientes físicos escolares tienen un impacto en el comportamiento, incluyendo la inclinación a participar en actividad física (Lake y Towshend, 2006; Lake y Towshend, 2013).

En relación con el género, se han encontrado que las barreras los impactan en forma diferente, lo que ha conducido a sugerir diferentes estrategias a la hora de promover la actividad física en el entorno del recreo escolar. Adicionalmente, es importante recalcar que la influencia de los factores ambientales sobre la actividad física durante el recreo difiere de la cultura y de los estilos de vida de los países, según estudios cualitativos desarrollados en diferentes culturas

Revista MHSalud@ (ISSN: 1659-097X) Vol. 13. No. 1. Setiembre-Enero, 2016 
(Ishii et al., 2014; Haug, Torsheim, Sallis y Samdal, 2010), lo que hace necesario indagar estos factores en el entorno costarricense.

Este estudio utilizó un diseño cualitativo, que es la forma reconocida para entender las percepciones de los niños y niñas con respecto a los factores ambientales y físicos que afectan la actividad física durante el recreo, lo que hace de este tipo de investigación, la forma idónea para obtener los insumos requeridos para el desarrollo de intervenciones y estrategias destinadas a promover el juego activo y, en general, la actividad física (Parrish, Yeatman, Iveson y Russell ,2012).

El modelo ecológico guio el componente cualitativo de esta investigación. Este modelo establece que la realidad es influenciada desde múltiples niveles que operan desde la parte interna del individuo y de factores externos como el apoyo social, la parte organizativa del entorno, las políticas públicas y el entorno comunal (Ward, Saunders y Pate, 2007). Debido a que los niveles de actividad física de los niños durante el recreo se ven afectados por múltiples factores, esta investigación se propuso entender los factores que determinan la actividad física en los recreos escolares desde la perspectiva de los niños, usando un acercamiento ecológico cualitativo.

\section{Procedimientos}

\section{Participantes}

La muestra contempló 50 niños y 40 niñas de entre 7 y 10 años de al menos un grupo de cada nivel del primer ciclo de una escuela pública urbana de la provincia de Heredia. El centro educativo de donde provienen los niños y niñas presenta un horario ampliado de 7 a.m. a 2 p.m. Cada día, los estudiantes tienen 60 minutos de receso, divididos en tres recreos. En general, el recreo del almuerzo es el más largo, siendo de 30 minutos.

La muestra respondió al muestreo típico: la orientadora seleccionó a los grupos participantes, siguiendo el criterio de pertenecer al primer ciclo de educación primaria (Patton, 2002). Adicionalmente, los grupos seleccionados se dividieron en 4 grupos focales.

\section{Instrumentos}

Para la conducción de los grupos focales, se utilizó un cuestionario con preguntas abiertas a los estudiantes de los grupos participantes. Este cuestionario se construyó con base en la revisión bibliográfica sobre la temática de recreos, los objetivos propuestos en la investigación y la experiencia de los investigadores, siguiendo el modelo socioecológico (Ward et al. 2007).

\section{Diseño de la investigación}

Para llegar a conocer cómo las barreras y los elementos que median para realizar actividad física son percibidos por los escolares, este estudio utilizó la metodología cualitativa, poniendo 
especial énfasis en los grupos focales. Los grupos focales han demostrado ser un método eficaz para reunir una amplia gama de puntos de vista y opiniones sobre un tema determinado, especialmente en los niños (Horner, 2000; Darbyshire, MacDougall y Schiller, 2005).

\section{Procedimientos}

Una vez que el estudio fue aprobado por el Ministerio de Educación y se contó con la anuencia del Director de la institución educativa, se obtuvo el consentimiento de los padres de las participantes.

Cada sección del primer ciclo de educación primaria seleccionada fue dividida en cuatro grupos mixtos focales por la orientadora de la institución. Las sesiones del grupo focal fueron realizadas en un lugar privado de la institución durante las horas de clase y fueron conducidas por el investigador con la ayuda de la orientadora de la institución y la maestra de grado. La orientadora y la maestra grabaron y tomaron apuntes de las sesiones, con el fin de fortalecer el análisis posterior de la información y lograr la triangulación necesaria (Patton, 2002). Cada una de las sesiones duró de 30 a 40 minutos. En ellas, primero se explicó la actividad y luego siguió el periodo de las entrevistas grupales, con un final abierto para la intervención de alguna anécdota que alguno de ellos quisiera compartir.

Para la conducción de los grupos focales, se utilizó una entrevista semiestructurada usando preguntas generadoras que emanaron esencialmente del modelo socioecológico. Con la intención de hacer que los participantes se sintieran más a gusto, se inició con una actividad lúdica; seguidamente, se procedió a inicio del grupo focal, con cuatro niveles de preguntas: preferencias de actividades de los niños en los recreos, factores que motivan a los niños a participar en el juego activo, factores que limitan el juego activo de los niños y factores que facilitan el juego activo de los niños.

\section{Análisis de la información}

En aras de garantizar la triangulación en la obtención de los datos, la información recopilada fue transcrita por el investigador, con la ayuda de la orientadora y la maestra de grado, después de la realización de cada grupo focal (Okuda-Benavides y Gómez-Restrepo, 2005). Una vez que se tuvo una descripción de la información, se procedió a realizar la codificación de la información siguiendo el análisis abierto, el axial y la codificación selectiva (Pitney y Parker, 2009), con el fin de poder definir las categorías de análisis de la información. En cada fase del análisis, las categorías determinadas se discutieron con el equipo de la escuela. Adicionalmente a la triangulación de investigadores, se utilizó la triangulación por teorías, utilizando el modelo ecológico que contempla los constructos de teorías múltiples, con las cuales se desarrollaron las categorías de intrapersonal, interpersonal, apoyo social, organizativo y de políticas (Ward et al., 2007). 


\section{Resultados}

Cinco categorías principales de análisis fueron definidas a la hora de explicar los resultados obtenidos con los niños y niñas durante el recreo escolar: el factor individual, el entorno social, el medio físico, los factores de la organización y de políticas escolares, siguiendo los planteamientos de una visión ecológica (Ward et al., 2007).

\section{Nivel individual}

En general, un $80 \%$ de los niños y niñas expresaron que las actividades preferidas de ellos durante el recreo fueron las que involucraban perseguirse mutuamente; sin embargo, los grupos de participantes que manifestaron más complacencia al jugar los juegos de "la anda" y "policías y ladrones", fueron los de primer y segundo grado. Los niños de tercer grado indicaron su afición por el futbol, mientras que las niñas mencionaron el caminar y hablar como las actividades preferidas.

En los niños de primero y segundo, el ser bueno no fue expresada como una limitante; sin embargo, en el nivel de tercero, el ser bueno jugando les garantiza mejenguear siempre. Esto se aprecia en el fragmento siguiente:

Entrevistador: Bueno, ¿por qué el futbol es uno de tus favoritos?

Rigo: Porque yo soy bueno en eso.

Entrevistador: Entonces, ¿y por qué ser bueno es tan importante?

Rigo: Es fácil, porque si eres bueno usted sabe cómo patear el balón, ya sabes cómo conseguir una falta y esas cosas. Y todos desean ser buenos en la mejenga.

Se hace evidente que el futbol ocupa un lugar importante en todos los niveles en primer ciclo, cuando se trata de los niños, sobre todo con los compañeros de la sección. Por ejemplo, pablo muere por el futbol y piensa que "hoy es día de revancha contra el equipo que nos ganó en el primer recreo". Aunque hay una gran aceptación por el futbol por parte de los niños, un 40 $\%$ de los niños no se involucran durante todos los días de la semana, principalmente porque no son parte del grupo de "mejengueros".

Por otro lado, de gran preocupación para los niños son los recreos tan cortos que a veces solo les permiten ir al baño y comer algo rápido. No queda tiempo para jugar. En este sentido, Jorge expresó: "nunca me da tiempo para comer algo en los recreos", y Maikol agregó: "prefiero jugar que comer".

Aunque el tener destrezas o ser bueno, como los niños lo mencionan, significa un elemento motivador para jugar y sentirse exitoso cuando practican deportes, no es la generalización, ya 
que cuando se refiere a jugar "policías y ladrones" o "la anda" se democratiza la participación y a la mayoría de niños y niñas les gusta participar. En los juegos de perseguir, el agente motivador expresado por los niños y niñas de primero y segundo es la sensación de disfrute. En relación con esto, Mario comentó: "se siente bien cuando logro agarrar a mis compañeros"; pero también el ser perseguido les imprime a los niños una sensación de vértigo, como expresó Jaime: “nadie me ha podido agarrar durante todos los recreos".

El siguiente intercambio ilustra la sensación de disfrute como un elemento importante en la participación:

Brian: Bueno, se siente bonito cuando usted puede atajar la bola.

Entrevistador: ¿Usted puede atajar la bola cuando la tiran a ras del suelo? ¿Qué parte se siente bien?

Brian: Yo me tiro como Keilor. Es mejor jugar con los compañeros porque usted tiene un mejor juego y disfrutamos más.

\section{Nivel interpersonal}

Dentro la esfera de los juegos de perseguir, muchos niños muestran un nivel de libertad que la escuela no les ofrece en la parte académica, sobre todo cuando los niños ven a sus maestras como gendarmes. Para ellos, las niñas limitan mucho la actividad de ellos. Muestra de esto son las expresiones de algunos de los niños: "queremos hacer lo que nos gusta, pero las niñas a veces nos quitan las bolas" o "la niña' nos castiga cuando no nos portamos bien, pero al final terminamos jugando en alguno de los recreos". Sin embargo, el rol de las maestras cambia cuando se trata de las mujeres, ya que las niñas se relacionan más con las maestras en los recreos. La sensación de los niños de ver el recreo como el espacio de liberación y de realización de lo que les gusta, sin restricciones, es uno de los elementos que se expresaron en los grupos focales en los niños, esto a pesar de que los más pequeños ven a las maestras como las protectoras de los niños menores.

\section{Preferencias de juego en las niñas o los niños}

Las niñas manifestaron que a ellas les gusta actividades menos vigorosas o sedentarias que involucran el grupo social de amigas, como caminar y hablar por las instalaciones de la escuela; sin embargo, un $54 \%$ de las niñas de primer grado se mostraron atraídas por juegos de perseguir como contagio y manifestaron que, cuando se presenta la oportunidad, ellas participan. La actividad que sigue teniendo vigencia en las niñas de primer ciclo es el salto de la cuerda y pareciera que, de contar con los mecates, las niñas se involucrarían. Siguiendo la línea de las actividades que les gusta más a las niñas en la escuela, Lucía expresó a cabalidad lo

1 En Costa Rica, se le denomina "la niña" a la maestra de Escuela. 
que las niñas en general piensan: "Lo que más me gusta es caminar y hablar con mis amigas".

A diferencia de las niñas, los niños expresaron preferencia por actividades en grandes grupos y juegos informales de futbol, sin olvidar los juegos clásicos de perseguir. Cuando de los niños de primero y segundo se trata, Martín expresó: "Apenas tocan el timbre todos corremos para aprovechar el recreo mejengueando".

Para establecer si los niños percibieron ser influenciados por lo que jugaron sus amigos, se les preguntó a los estudiantes si sus amigos juegan los mismos juegos como lo hicieron en recreo y el almuerzo. Aproximadamente la mitad de los niños dijeron que jugaron los mismos juegos que sus amigos. Sin embargo, algunos niños identificaron la preferencia por hacer algo diferente, condicionado a si sus amigos les gustaba el mismo juego. "A veces me gusta jugar balón con la mano pero no lo hago, así que solo estoy jugando con ellos”, dijo Marvin.

En los casos en que los niños y las niñas expresaron actividades que realizan conjuntamente, mencionaron los juegos de "la anda" (infectado o policías y ladrones) y otros niños mencionaron el salto de la cuerda. Cuando los niños interactúan saltando la cuerda con las niñas, ellas los ven con recelo, ya que los niños no toman turnos e interrumpen el juego. En relación con esto, Marcos dijo: "A mí me gusta saltar mecate cuando la niña participa"; en forma diferente, Marcela expresó: "Los niños vienen saltan y se van, no tienen paciencia para esperar el turno" o lo que dice Roció: "Ellos se cuelan".

Por otro lado, los mayores ven a los más pequeños como obstáculos que interrumpen la mejenga y se ponen en el camino de ellos. "Generalmente, tenemos que dejar de jugar hasta que los corremos", expresó Martín.

El uso de teléfonos para jugar videojuegos es una actividad popular en los niños y las niñas, especialmente entre los que no expresaron participar en los juegos. Para muchos, los aparatos electrónicos significan el poder realizar algo en forma individual, en especial en las aulas durante los recreos. Sin embargo, algunos niños que usan aparatos electrónicos expresaron que les gustaría jugar, pero ellos se consideran que no son buenos. César dijo: "Hay juegos que no juego durante los recreos, pero lo haría si fuera bueno jugando, como mis compañeros".

\section{Nivel organizativo}

Aunque es conocido, los niños y niñas se ajustan al espacio con que cuentan; sin embargo, muchos de los niños expresaron que necesitan correr para proteger el espacio de la mejenga, utilizando cada segundo para mantener este espacio. El grupo de Juan diseñó una estrategia para mantener el espacio. Claro dice Juan: "Yo tengo que correr apenas tocan para salvar el espacio, pero a veces voy al baño para estar más cerca del planché".

El no poder contar con un espacio de juego no representa ningún problema para los niños y las niñas, ya que ellos juegan en cualquier espacio, uno de ellos expresó: "La anda es lo que más me gusta jugar en el recreo grande y siempre jugamos hombres contra mujeres". Aunque 
muchos expresan pocas limitaciones de espacio, ellos utilizan los corredores y cualquier espacio para jugar. Luis piensa que "los corredores me ayudan a escaparme de las que me persiguen, ya que hay muchos niños". Evidentemente los espacios en las escuelas urbanas son reducidos, pero los niños se valen de cualquier espacio para jugar, como lo dice Jaime: "A veces jugamos futbol en el mismo lugar, mientras otros juegan".

En el caso de las niñas, los espacios preferidos no entran en competencia con otros u otras, ya que ellas expresaron que les gustan los lugares que les permita mayor privacidad. Maritza expresó: "Mis amigas y yo caminamos y hablamos lejos de donde juegan futbol". También, en general muchas niñas $\mathrm{y}$, en algunos casos, los niños más pequeños se sienten amenazados por los niños mayores, ya que ellos podrían invadir o molestarlos. En relación con esto, Luis dijo: "ellos vienen y nos patean la botella" o como expresó Víctor: "Los mayores se creen los jefes de la escuela".

La escuela es un ambiente social que les ofrece a los niños y niñas el establecimiento de niveles o jerarquías entre los niños mayores y los pequeños; sin embargo, presenta a las maestras como el ente que protege a los más pequeños. Aunque las maestras asumen un rol pasivo en los recreos, por lo general, se encargan de mantener la tranquilidad. "Me gusta cuando la niña cuida recreos para jugar mecate", dijo Silvia, o "con la niña se van los matones". Sin embargo, para César la niña es la inquisidora cuando: "No me deja salir a recreo hasta que termine el trabajo". Los niños sienten que las maestras pueden ser un obstáculo para algunos de ellos y que las reglas de ellas o de la escuela evitan que a veces ellos puedan jugar. Carlos expresó: "No nos dejan jugar trompos por la niña Tere, que le pasó un trompo cerca de la cara y se quejó". Muchas de las medidas que se toman en los recreos son basadas en temores de que alguien se lesione; sin embargo, los niños se sienten desprotegidos y limitados por las decisiones de dejar de hacer algo. "No nos dejan jugar futbol, pero nosotros jugamos con las botellas vacías".

El juego es tomado tan en serio por los niños que, al momento de conformar los equipos o grupos para jugar, a menudo hay algún desacuerdo. Una conversación entre el que condujo los grupos focales y tres niños reflejan esto:

Entrevistador: ¿Hay a menudo conflictos a la hora de jugar o formar los equipo?

Mario: Sí, en el campo de fútbol.

Beni: Casi todos los días.

Rafa: A menudo alguien lucha, pero no todos los días.

Moderador: ¿De qué discuten?

Beni: Si se trata de un gol o un tiro libre.

Luis: O balón con la mano.

Beni: Alex, él sólo quiere ganar. 
Los conflictos causados por los niveles de poder de los niveles superiores también se experimentan como una barrera importante para el juego durante el recreo. Algunos niños se sintieron maltratados por niños mayores que "destruyeron" su juego. En este sentido, Mario expresó: "Los de sexto pueden simplemente ser un poco molestos cuando vienen y dicen que tenían el campo de fútbol primero. Entonces tenemos que encontrar una maestra que nos ayude".

\section{Politicas institucionales en los recreos}

Las barreras más frecuentemente mencionadas por niños para realizar la actividad física en la escuela son: la falta de instalaciones, la falta de espacio para jugar y las pausas breves, pero, sobre todo, que no les permiten mejenguear. Las escuelas a menudo tienen muy bien estructurado el cuido de los recreos escolares por parte de las maestras, con un énfasis en evitar que los niños se maltraten; sin embargo, los niños sí manifiestan cierta persecución con respecto a no permitirles jugar algunos juegos. Carlos expresó que "ellos se esconden de la niña Xinia para que no les quite la bola". Pero también las políticas y lugares de juego no son parte de las preocupaciones de la administración escolar y depende algunas veces de la maestra el ignorar alguna disposición que prohíbe jugar bola y les permite jugar a los niños a sus anchas.

Las maestras enfrentan cotidianamente algún nivel de desinterés de algunos de los niños y niñas, en relación con el rendimiento académico, lo que ha hecho que muchas de ellas utilicen los recreos para lograr motivar grupalmente a los mejengueros de la clase. Al respecto, la niña Susana expresó: "Los niños que no terminan se tienen que quedar en el recreo terminando". Pareciera que esta estrategia se presenta como algo común, lo cual es posiblemente criticable, pero es la forma en que algunas maestras logran encauzar a los niños y niñas con la parte académica.

Evidentemente, las maestras han manifestado la importancia del recreo para todos los niños; sin embargo, pareciera que en la práctica ellas no lo ven como el espacio en que los niños logran expresarse corporalmente y prepararse para un mejor rendimiento académico. La niña Marta refleja lo que las maestras del grupo focal expresaron: "Claro que es importante que corran y salten, para que así se tranquilicen". Las políticas a nivel de recreo para que los niños y niñas jueguen es algo que no existe en esta escuela; sin embargo, las maestras piensan que se sería útil señalar lugares para jugar o darles algún tipo de material.

\section{Discusión}

Este estudio ha pretendido tener un mejor entendimiento de los factores que determinan la actividad física en el entorno escolar desde la perspectiva de los niños y las niñas. Tomando como referencia el modelo socioecológico (Ward et al., 2007), cinco categorías principales de análisis fueron definidas a la hora de explicar los resultados obtenidos con los niños y las niñas durante el recreo escolar: el factor individual, el entorno social, el medio físico y los factores de la organización escolar. Este análisis ha revelado que las influencias del entorno social y 
organizacional han sido percibidas como las de mayor peso a la hora de involucrarse en actividad física, durante los recreos escolares por parte de los niños y niñas de este estudio, lo cual hace indispensable que los programas o intervenciones escolares en la promoción de la actividad física contemplen, con mayor énfasis, estos factores. Recientemente, autores como Ishii et al. (2014) han enfatizado la necesidad de replantearse el papel de las escuelas para que lideren la promoción de la salud física y la prevención contra la obesidad. Estos autores recalcan los elementos de apoyo social y el entorno físico. El entender, desde la perspectiva de los niños y niñas, que los niveles que perciben con mayor influencia para realizar actividad física son el entorno social y físico indica que las estrategias en la promoción de la salud física que contribuyan a la salud integral de los escolares deben contemplar el trabajar para eliminar esas barreras y contribuir a incrementar la percepción de ellos hacia entornos que propician movimiento.

En el factor individual, al igual que ha sido reportado en diferentes investigaciones, para los niños y niñas de esta investigación el recreo es parte importante del día para ellos; sin embargo, este momento ya no se percibe tan libre para poder organizar sus propios juegos, jugar en equipos, correr, perseguirse unos a otros y saltar la cuerda, ya que ellos perciben limitantes que los encasillan cada vez más en conductas sedentarias. En comparación con el resto de la jornada escolar, el recreo sigue siendo el tiempo en el que los niños tienen más posibilidades para elegir lo que quiere hacer y con quién. Para muchos niños, el recreo es su parte preferida del día y los niños de este estudio así lo han manifestado. Pareciera que el recreo escolar sigue siendo un espacio de disfrute, como también lo han reportado otros estudios (Mays, Graber y Daum, 2012; Sallis, Prochaska y Taylor,2000)

Un elemento importante de recalcar son las preferencias de las actividades de niños y niñas en el recreo escolar, donde los niños prefieren involucrarse en actividades de moderada intensidad a vigorosa, lo que corresponde a datos similares de otros estudios (Hallal et al. 2012; Sallis et al., 2000; Trost, Pate, Freedson, Sallis y Taylor, 2000). También se ha reportado en diferentes estudios que los niños se involucran más en actividades de moderada intensidad que las niñas, independientemente si se cuenta con las condiciones ambientales apropiadas (Santos, Guerra, Ribeiro, Duarte y Mota,2003; Trost et al., 2002). Por otra parte, el tiempo de juego representa el principal contexto en el que las niñas y los niños tienen la misma oportunidad de ser físicamente activos diariamente (Skau, Tjornhoj-Thonsen, Schipperijn y Troelsen et al., 2014; Ridgers, Stratton y Fairclough, 2006; Sarkin, McKenzie y Sallis, 1997); sin embargo, se debe considerar que ellas no se involucran espontáneamente en actividades de moderada intensidad, cuando se comparan con los niños.

Aunque las niñas de este estudio reportaron que las actividades preferidas son las relaciones sociales que involucran el hablar y caminar en grupos pequeños, ellas expresaron el deseo de involucrarse en actividades físicas, si existieran mejores condiciones. En relación con esta percepción que limita el ser más activas a las niñas, este es un elemento básico que debe ser atendido para garantizar el incremento de la actividad física en el entorno de los recreos escolares. No obstante, se requiere procesos de investigación adicionales para determinar las preferencias de las niñas en 
referencia a la actividad física y al espacio disponible. Adicionalmente, existe toda una tendencia de que si existen las condiciones ambientales apropiadas, el recreo ofrece el mayor potencial diario para que las niñas incrementen sus posibilidades de ser más activas (Mota et al, 2005).

En este estudio, los participantes que utilizaron artefactos electrónicos en la escuela percibieron que el uso de estos era una barrera para participar en conductas activas, lo que podría ser un factor que interfiere en el desarrollo motor normal, impidiendo el involucramiento exitoso en actividad física durante el recreo, como lo ha establecido Ridgers et al. (2012). Esta nueva barrera que limita a muchos niños y niñas para que sean activos físicamente debe ser atendida. Investigaciones adicionales son necesarias para explorar el impacto de esta nueva barrera.

En el área interpersonal, los estudiantes en este estudio perciben que el rol de las maestras y de los administradores durante el recreo es pasivo; no obstante, incluso también expresaron que ellos asumen posiciones de impedir la actividad física en los recreos o usan este espacio para disciplinar a los niños, lo cual ha sido criticado fuertemente por Ramstetter, Murray y Garner (2010). A pesar de lo anterior, en las entrevistas con las maestras hay una aceptación generalizada por parte de ellas de la importancia del recreo en las áreas cognitiva, social, emocional y física.

Aunque en este estudio la labor de la maestra durante el recreo se percibió como una barrera, el aumento en la supervisión de la maestra, en particular cuando ellas participan en el juego, brinda mayor satisfacción y participación por parte de las niñas, propiciando una reducción de conflictos y menos dominación por parte de los niños, como lo han expresado varios estudios (Sallis et al., 2001; Ozdemir y Yilmaz, 2008).

Al ser considerada la falta apoyo social como una barrera importante, es necesario mencionar que este nivel de influencia es considerado más importante que el personal (Mays et al., 2012), lo que hace de suma importancia entender que el recreo es una extensión de la experiencia académica de las aulas, por lo que se recomienda que los niños y niñas tengan las condiciones físicas y el apoyo social necesario para que puedan obtener el máximo beneficio de ambientes que estimulan el juego y la actividad física, así como la mayor cantidad de posibilidades para que jueguen (Ickes, Ervin y Beighle, 2013).

En lo referente a conflictos y ambiente, la percepción de los niños de este estudio en relación con el matonismo fue manifestada como la lucha constante de los niños pequeños con los mayores por el espacio más apropiado para jugar futbol. La intimidación podría tener un efecto sobre los niveles de actividad de juegos de los niños y especialmente de las niñas. Veitch (2007) sitúa esta barrera como una de las razones mayores para no participar en actividades físicas en el tiempo libre de los niños menores. Como una forma de evitar el matonismo o la amenaza de niños mayores, ha sido recomendado que las instituciones demarquen las zonas donde los niños y niñas más pequeños pueden jugar en los recreos, como una de las primeras medidas relacionadas con políticas de convivencia pacífica en el entorno escolar (Ridgers et al., 2012).

Estudios en Inglaterra y Australia (Parrisch et al., 2012; Ridgers, Fairclough y Stratton, 2010) han mostrado que la percepción de seguridad que ofrece el ámbito escolar en el recreo 
para los niños y niñas no presenta diferencias; sin embargo, los hallazgos de este estudio sí muestran que las niñas se sienten intimidadas en ambientes donde no se presenta la supervisión o presencia de las maestras. Este hecho se podría explicar por el hecho de que las niñas expresaron que utilizaron en forma diferente el espacio, inclinándose más a socializar, conforme crecen.

Estos hallazgos sugieren que un acercamiento intervencional que se focalice en el mejoramiento del equipo, las facilidades y la organización del recreo en el entorno escolar podría ser una forma efectiva para incrementar el juego y el movimiento en los niños y niñas.

En cuanto a los reglamentos o regulaciones institucionales durante el recreo (disposiciones administrativas), los niños perciben oportunidades y amenazas para su participación durante el recreo. Ellos entienden que muchas de las reglas en el recreo responden al temor de la administración escolar con respecto a asuntos de seguridad; sin embargo, los niños muchas veces se quejan de medidas extremas como el prohibir la mejenga de fútbol. Esto lo perciben como la incomprensión de los adultos, lo cual genera una reacción de rechazo y de transgredir la disposición, jugando con cualquier cosa que ruede. Estos hallazgos son congruentes con investigaciones cualitativas que mencionan factores que podrían ser considerados como barreras para realizar actividad física o jugar durante los recreos. Estos factores son: las destrezas básicas, la estación lluviosa, la disponibilidad y las características de los espacios al aire libre y las características de la escuela; todos elementos básicos que hay que tomar en cuenta a la hora de desarrollar estrategias para activar a los niños y niñas durante el recreo escolar (Lake y Townshend, 2013).

Dentro de los factores ambientales, el nivel de influencia del espacio fue percibido como una barrera importante para la realización de la actividad física en este estudio, lo que también se ha reportado en otros estudios (Stanley et al. 2012; Lake y Townshend, 2013). Esto también es apoyado por los hallazgos de otros estudios cuantitativos en los que se demuestra que entre más espacio de juego, más actividad física se realizó en el recreo con niños escolares en Australia y Canadá (Veitch, 2007; Humberth et al., 2006). Además, estas investigaciones reportan que los conflictos se relacionan con situaciones de organización de los equipos, actividades y disposiciones institucionales.

En la categoría de redes de amigos y amigas, los niños que reportaron ser más activos en este estudio percibieron que la actividad física estaba determinada por su grupo de amigos. Entre más cerca y más fuerte la conexión de amistad, mayor influencia existe (Mays et al 2012; Duncan, Duncan y Strycker, 2000; Lin, 2001). Pero, más importante, son los indicios de que los niños deciden su participación en actividad física sobre las acciones y mensajes que reciben de parte de sus amigos, cuando se trata de actividades físicas no estructuradas (Jago et al. 2005).

Sobre el ambiente y en consonancia con los modelos socioecológicos (Ward et al, 2007), los niños y niñas de este estudio percibieron los espacios ambientales como determinantes para la realización de actividad física en los recreos. En este estudio, los niños y las niñas sintieron que la falta de espacio era una barrera, pero esto fue más evidente en las niñas, posiblemente porque los niños tienden a dominar las áreas principales del patio de la escuela (Skau et al., 2014; Boyle, Marshall y Robeson, 2003). Al respecto, Ridgers et al. (2010), no encontraron ninguna 
asociación significativa entre el tamaño de la zona de juego y la actividad física entre los niños y las niñas durante el recreo.

\section{Conclusiones}

El entendimiento de las percepciones de los niños sobre las barreras y los mediadores para realizar actividad física durante los recreos de los participantes refuerza el hecho de que la conducta de actividad física está influenciada por una interacción compleja de factores de diferentes niveles, incluyendo los intrapersonales, los sociales, el ambiente físico, la organización y las políticas escolares

Se puede concluir que los niños y niñas percibieron barreras ambientales (físicas, sociales, institucionales) y factores personales que afectan los niveles de actividad física en el recreo escolar en forma diferente. Específicamente, los factores identificados por los participantes en este estudio fueron: los conflictos interpersonales, la falta de espacio en la institución, la falta de apoyo social, la falta de instalaciones de juego y el uso de dispositivos electrónicos. Estas barreras son percibidas en forma diferente por los niños y niñas, lo que hace necesario la utilización de diferentes acercamientos para promover la actividad física dependiendo del género.

El acercamiento ecológico utilizado en esta investigación ha indicado la importancia de utilizar múltiples niveles de influencia, así como el rol del ambiente, al determinar la percepción de los niños y las niñas acerca de las conductas de movimiento de ellos en el entorno escolar. En este sentido, se debe poner especial énfasis en la percepción de las barreras de apoyo social y ambiente físico, que son los factores que han evidenciado ser los que presentan el mayor peso a la hora de propiciar conductas de actividad física entre la población escolar de este estudio.

Se recomienda que la actividad física en el recreo escolar sea promovida a través de una combinación de acciones que aborden las barreras dentro de una visión multinivel, utilizando el nivel individual, social, ambiental y organizativo. Esto implica el uso de un enfoque socio-ecológico.

\section{Referencias bibliográficas}

Biddle, S.J.H. \& Asare, M. (2011). Physical activity and mental adolescents: a review of reviews. British Journal of Sports Medicine, 45 (11), 886-895. http://dx.doi.org/10.1136/ bjsports-2011-090185

Boyle, D.E., Marshall, N.L. \& Robeson, W. (2003). Gender at play-Fourth-grade girls and boys on the playground. American Behavioral Scientist, 46 (10), 1326-1345. http://dx.doi. org/10.1177/0002764203046010004

Boreham, C. \& Riddoch, C. (2001). The physical activity, fitness and health of children. Journal of Sports Science, 19 (12), 915-929. http://dx.doi.org/10.1080/026404101317108426 
Buchan, D., Ollis, S., Thomas, N. \& Baker, J. (2012). Physical Activity Behaviour: An Overview of Current and Emergent Theoretical Practices. Journal of Obesity, 34, 1-11. http://dx.doi. org/10.1155/2012/546459

Darbyshire, P., MacDougall, C. \& Schiller W. (2005). Multiple methods in qualitative research with children: more insight or just more? Qualitive Research, 5 (4), 417-436. http://dx.doi. org/10.1177/1468794105056921

Duncan, S.C., Duncan, T.E. \& Strycker, L.A. (2000). Risk and protective factors influencing adolescent problem behavior: a multivariate latent growth curve analysis. Annals of Behavioral Medicine, 22 (2), 103-109. http://dx.doi.org/10.1007/BF02895772

Floriani, V. \& Kennedy, C. (2008). Promotion of Physical Activity in Children. Curr. Opin. Pediatric, 20, 90-95. http://dx.doi.org/10.1097/MOP.0b013e3282f3d9f9

Hallal, P.C., Andersen, L.B., Bull, F.C., Guthold, R., Haskell, W. \& Ekelund, U. (2012) Global physical activity levels: surveillance progress, pitfalls, and prospects. The Lancet, 380, 247-257.

Haug, E., Torsheim, T., Sallis, J.F. \& Samdal, O. (2010). The characteristics of the outdoor school environment associated with physical activity. Health Education Research, 25 (2), 248-256. http://dx.doi.org/10.1093/her/cyn050

Horner, S.D. (2000). Using focus group methods with middle school children. Research in Nursing and Health, 23 (6), 510-517. http://dx.doi.org/10.1002/1098-240X(200012)23:6<510::AIDNUR9>3.0.CO;2-L

Humbert, M.L., Chad, K.E., Spink, K.S., Muhajarine, N., Anderson, K.D., Bruner, M.W., Girolami, TM., Oknokon, P. \& Gryba CR.(2006). Factors that influence physical activity participation among high- and low-SES youth. Qualitative Health Research, 16 (4), 467483. http://dx.doi.org/10.1177/1049732305286051

Ickes, M., Erwin, H., \& Beighle, A. (2013). Systematic Review of Recess Interventions to increase Physical Activity. Journal of Physical Activity and Health, 10, 910-926.

Ishii, K., Sato, M., Shibata, A. \& Oka, K., (2013). Perceived School Physical Activity Environment and School Physical Activity among Japanese Elementary School Children. Japan Journal of Human Growth and Development Research 59, 1-11. Recuperado de: https://www.jstage.jst.go.jp/article/hatsuhatsu/2013/59/2013_1/_pdf

Ishii, K., Shibata, A., Sato, M. y Koichiro Oka, K. (2014). Recess Physical Activity and Perceived School Environment among Elementary School Children. Journal of Environmental Research and Public Health, 11, 7195-7206. http://dx.doi.org/10.3390/ijerph110707195 
Janz, K.F., Burns, T.L. \& Levy, S.M. (2005). Tracking of Activity and Sedentary Behaviors in Childhood: The Iowa Bone Development Study. American Journal of Preventive Medicine, 29 (3), 171-178. http://dx.doi.org/10.1016/j.amepre.2005.06.001

Jago, R., Baranowski, T., Zakeri, I. \& Harris M. (2005). Observed environmental features and the physical activity of adolescent males. American Journal of Preventive Medicine, 29(2), 98-104. http://dx.doi.org/10.1016/j.amepre.2005.04.002

Mays, A., Graber, K. \& Daum, D. (2012). Children's recess physical activity: Movement patterns and preferences. Journal of Teaching in Physical Education, 31, 146-162. Recuperado de http://journals.humankinetics.com/jtpe-back-issues/jtpe-volume-31-issue-2-april/ childrenrsquos-recess-physical-activity-movement-patterns-and-preferences

Mota, J., Silva, P., Santos, M., Ribeiro, J., Oliveira, J. \& Duarte, J. (2005). Physical activity and school between the sexes and the relationship between children playground physical activity and habitual physical activity. Journal of Sports Sciences, 23 (3), 269-275. http:// dx.doi.org/10.1080/02640410410001730124

Lake, A.A. \& Townshend, T.G. (2006). Obesogenic environments: exploring the built and food environments. Perspectives in Public Health, 126 (6), 262-267. http://dx.doi. org $/ 10.1177 / 1466424006070487$

Lake, A.A \& Townshend, T.G. (2013). Exploring the built environment, physical activity and related behaviors of young people attending school, college and those not in employment. Journal of Public Health, 35 (1), 57-66. http://dx.doi.org/10.1093/pubmed/fds059

Lin, N. (2001). Social Capital: A Theory of Social Structure and Action. http://dx.doi.org/10.1017/ CBO9780511815447

Okuda-Benavides, M. \& Gómez-Restrepo, C. (2005). Métodos en investigación cualitativa: triangulación. Revista Colombiana de Psiquiatría, XXXIV, 118-124. Recuperado de http:// $\underline{\text { www.redalyc.org/articulo.oa?id=80628403009 }}$

Ozdemir, A. \& Yilmaz, O.(2008). Assessment of outdoor school environments and physical activity in Ankara's primary schools. Journal of Environmental Psychology, 28 (3) . 287300. http://dx.doi.org/10.1016/j.jenvp.2008.02.004

Parrish, A., Yeatman, H., Iveson,D \& Russell, K. (2012). Using Interviews and Peer Pairs to Better Understand how School environments Affect Yong Children Playground Physical Activity Levels: a Qualitative Study. Health Education Research, 27, 269-280.

Patton, M.Q. (2002). Qualitative research and evaluation methods (3rd ed.). Thousand Oaks, CA: Sage. 
Pawlowski, C., Shipperijn, J. \& Troelsen, J. (2014). Barriers for Recess Physical Activity: A Gender Specific Focus group Exploration. BMC Public Health, 14, 639. Recuperado de: http://bmcpublichealth.biomedcentral.com/articles/10.1186/1471-2458-14-639

Pitney, W \& Parker, J. (2009). Qualitative Research in Physical Activity and the Health Professions.Champaign, IL: Human Kinetics.

Ramstetter, C.L., Murray, R. \& Garner, A.S. (2010). The Crucial Role of Recess in Schools. Journal of School Health, 80 (11), 517-526. http://dx.doi.org/10.1111/j.1746-1561.2010.00537.x

Ridgers, N.D., Salmon, J., Parrish, A.M., Stanley, R.M. \& Okely, A.D. (2012) Physical activity during school recess a systematic review. American Journal of Preventive Medicine, 43 (3), 320-328. http://dx.doi.org/10.1016/j.amepre.2012.05.019

Ridgers, N.D., Fairclough, S.J. \& Stratton, G. (2010). Variables associated with children's physical activity levels during recess: The A-CLASS project. International Journal of Behavioral Nutrition and Physical Activity, 7 (74), 1-8. http://dx.doi.org/10.1186/1479-5868-7-74

Ridgers, N.D., G. Stratton, \& S.J. Fairclough. (2006). Physical activity levels of children during school playtime. Sports Med, 36 (4), 359-371.

Sallis, J. F., Prochaska, J. J., \& Taylor, W. C. (2000). A review of correlates of physical activity of children and adolescents. Medicine and Science in Sports and Exercise, 32, 963-975. http://dx.doi.org/10.1097/00005768-200005000-00014

Sallis, J.F., Conway, T.L., Prochaska, J.J., McKenzie, T.L., Marshall, S.J \& Brown, M. (2001). The association of school environments with youth physical activity. American Journal of Public Health, 91 (4), 618-620

Salmon, J. \& Timperio, A. (2007). Prevalence, trends and environmental influences on child and youth physical activity. Med Sport Sci, 50, 183-199. http://dx.doi.org/10.1159/000101391

Santos, P., Guerra, S., Ribeiro, J. C., Duarte, J. A., \& Mota, J. (2003). Age -and gender- related physical activity: A descriptive study in children using accelerometry. Journal of Sports Medicine and Physical Fitness, 43 (1), 85-89.

Sarkin, J. A., McKenzie, T. L.,\& Sallis, J. F. (1997). Gender differences in physical activity during fifthgrade physical education and recess period. Journal of Teaching Physical Education, 17, 99- 106.

Skau, C., Tjornhoj-Thonsen, T., Schipperijn, J. \& Troelsen, J. (2014) Barriers for Recess Physical Actvity: a gender specific qualitative focus group exploration. BMC Public Health, 14, 639.

Stanley, R.M., Boshoff, K. \& Dollman, J. (2012). Voices in the playground: a qualitativeexploration of the barriers and facilitators of lunchtime play. Journal of Science and Medicine Sport, 15 (1), 44-51. http://dx.doi.org/10.1016/j.jsams.2011.08.002 
URL: http://www.revistas.una.ac.cr/mhsalud

Strong, W.B., Malina, R.M., Blimkie, J.R., Daniels, S.R., Dishman, R.K., Gutin, B.\& Hergenroeder, A.C. (2005). Evidence based physical activity for school-age youth. The Journal of Pediatrics, 146 (6), 732-737. 5.

Trost, S.G., Pate, R.R., Freedson, P.S., Sallis, J.F. \& Taylor, W.C. (2000). Using objective physical activity measures with youth: How many days of monitoring are needed? Medicine and Science in Sports and Exercise, 32, 426-431. http://dx.doi.org/10.1097/00005768$\underline{200002000-00025}$

Trost SG, Pate RR, Sallis JF, Freedson PS, Taylor WC, Dowda M \& Sirad J. (2002). Age and gender differences in objectively measured physical activity in youth. Medicine \& Science in Sports \& Exercise, 34(2), 350-355 http://dx.doi.org/10.1097/00005768-200202000-00025

Van Sluijs, E.M.F., McMinn, A.M. \& Griffin, S.J, (2007). Effectiveness of interventions to promote physical activity in children and adolescents: systematic review of controlled trials. British Medical Journal, 335, 703. http://dx.doi.org/10.1136/bmj.39320.843947.BE

Veitch, J. (2007). Children's perceptions of the use of public open spaces for active free-play. Children's Geographies, 5(4), 409-422. http://dx.doi.org/10.1080/14733280701631874

Ward, D., Saunders, R. \& Pate, R. (2007). Physical Activity Interventions in Children and Adolescents. United States: Human Kinetics.

Wareham, N.J., Van Siuijs, E.M.F. \& Ekelund, U. (2005). Physical activity and obesity prevention: a review of the current evidence. Proceedings of the Nutrition Society, 64 (2), 229-247. http://dx.doi.org/10.1079/PNS2005423

Wing, R.R., Goldstein, M.G., Acton, K.J., Birch, L.L., Jakicic, J.M., Sallis, J.F.Jr, Smith-West, D., Jeffery, R.W. \& Surwit, RS. (2001). Behavioral science research in diabetes: lifestyle changes related to obesity, eating behavior, and physical activity. Diabetes Care, 24(1), 117-123. http://dx.doi.org/10.2337/diacare.24.1.117

Recepción: 10 de febrero del 2016

Corrección: 11 de mayo del 2016

Aceptación: 11 de junio del 2016

Publicación: 30 de julio del 2016

1 El autor es Doctor en Educación por la Universidad Nacional de Costa Rica; Profesor de la Escuela de Ciencias del Movimiento Humano y Calidad de Vida en la Carrera de Promoción de la Salud Física y Especialista en Fisiología y Ejercicio. 